\title{
LITHOLOGIC INVERSION OF TOMOGRAPHIC DATA
}

\author{
Fechner,Th. and Dietrich, $P$. \\ Geological Institute \\ University of Tuebingen \\ Sigwarstr.10, D-72076 Tuebingen
}

\begin{abstract}
Subsurface heterogeneity, i.e. the spatial variability of porous rock properties such as porosity or hydraulic conductivity, plays an important role in the spreading of a solute in the subsurface. A few highly-conductive zones may dominate the overall flow regime. Due to drilling costs the number of boreholes drilled to investigate an area is limited leading to uncertainties in determining the hydraulic properties and in resolving structures relevant for subsurface solute movement. A detailed subsurface characterization is however of vital importance for clean up technologies: for instance the efficiency and effectiveness of a "funnel \& gate"-systems is dependent on its optimal positioning. It is shown, that high-resolution measurements such as seismic and electric tomography can be used to identify hydraulic subsurface structures. For the problem of inversion of hydraulic properties and related subsurface structures a new formalism based on multivariate statistics is proposed.
\end{abstract}

\section{Introduction}

During the era of the former German Democratic Republic, the name "Bitterfeld" stood for the chemical industry. After the unification of Germany it became obvious that an ecological wasteland was imminent in the region. Lignite mining and the enormous state-run chemical combines had polluted the air, water and soil. For this reason highest priority has been given to clean up measures and installation of environmental technology such as "funnel \& gate" systems. The identification of subsurface structures and their related hydraulic properties plays an important role if hydraulic clean up technologies such as "funnel\&gate" systems and "pump \& treat" techniques are applied for remediation of the groundwater. Just a few high permeable zones may dominate the overall flow regime. Subsurface structures and their related parameters may vary over several $\log$-scales in only a few metres. Boreholes drilled for investigations may therefore miss the hydraulic structures relevant for groundwater flow and transport. Tomographic methods such as seismic or electric tomography have proved to be valuable tools for mapping the subsurface with a high resolution between boreholes [1,2]. Nevertheless, the inversion of geophysical data into the hydraulic parameters of an aquifer is based upon certain assumptions and petrophysical models, which may not be valid under specific conditions at a site. Furthermore, a relation between acoustic and hydraulic properties or electric and hydraulic properties of aquifer sediments suffers from ambiguity [3]. An alternative approach to characterizing the petrophysical properties of sediments can be taken using different geophysical and hydraulic methods to overcome the interpretation limits and applying geostatistical inversion methods [4,5]. The inversion method presented in this paper is based on multivariate statistics, i.e. cluster analysis, which allows a statistical description of subsurface parameters [6]. The inversion formalism can be applied independently of a specific 
tomographic forward model. As an inversion result an estimate of lithologic parameters is obtained. At each subsurface grid point a parameter set (seismic velocity, electric conductivity, porosity etc.) clustered into different groups is generated. To set up the number of clusters hydrogeological a-priori information such as borehole data can be included to specify the different lithotypes most probable at the site and to invert the most likely type of sediment (silt, fine sand, gravel) together with the related geophysical parameters of seismic velocity and electric conductivity. In addition to a zonation of subsurface lithology average geophysical and hydraulic values for each cluster are assigned resulting in a "binary" image or lithogram, i.e. depending upon the number of chosen clusters or lithotypes.

\section{Tomographic measurements}

The tomographic measurements were carried out at a test-site near Bitterfeld, Germany, see fig. 1. The distance between boreholes $3 / 97$ and 4/97 was about $10 \mathrm{~m}$. Seismic tomography was carried out as crosshole measurements over a depth of $13 \mathrm{~m}$. In order to obtain a high resolution an electrical discharge source, i.e. a sparker source, was used to generate the seismic signals. The received signals contained high frequency components up to $3 \mathrm{kHz}$. The sparker source allowed highly reliable measurements. The source operated in borehole 4/97 at a spacing of one metre. A 6-channel hydrophone chain with $0.5 \mathrm{~m}$ interval was used to record the signals. About 600 first arrival times were analysed and inverted using a SIRT inversion algorithm.

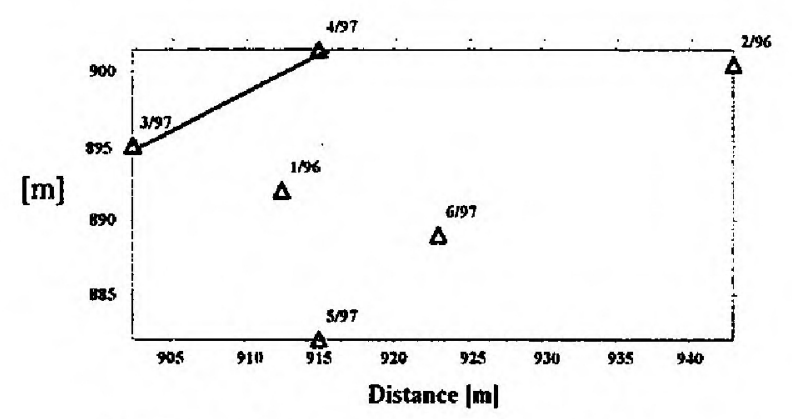

Fig. 1: Location of boreholes at the site

The electrical measurements were carried out in crosshole mode. The electrodes were mounted in boreholes $3 / 97$ and 4/97 in the gravel pack at an interval of one metre. Additionally, surface electrodes were placed in-line at a spacing of $1.5 \mathrm{~m}$. The measurements were carried out using multi-electrode equipment allowing a measuring speed of about $30 \mathrm{~s}$ to record the potentials at 94 electrode pairs. From the pole-pole measurements about 7.900 potentials were calculated using the principle of superposition. The potentials satisfied various quality measures. The inversion of the electrical data was carried out using the sensitivity analysis [7].

\section{Joint inversion of tomographic data}

The seismic tomogram shown in figure 2, indicates an almost horizontal layering of the sediments. A high velocity zone which ranges from $-10 \mathrm{~m}$ to $-12 \mathrm{~m}$ between borehole $3 / 97$ and $4 / 97$ is at the top of the tomogram. Furthermore, a low velocity layer with seismic velocity up to $1650 \mathrm{~m} / \mathrm{s}$ is shown at $-16 \mathrm{~m}$. The layer broadens in the direction of borehole $4 / 97$. The intermediate zones consisting of coarse sediments have seismic velocities of about $1750 \mathrm{~m} / \mathrm{s}$. 


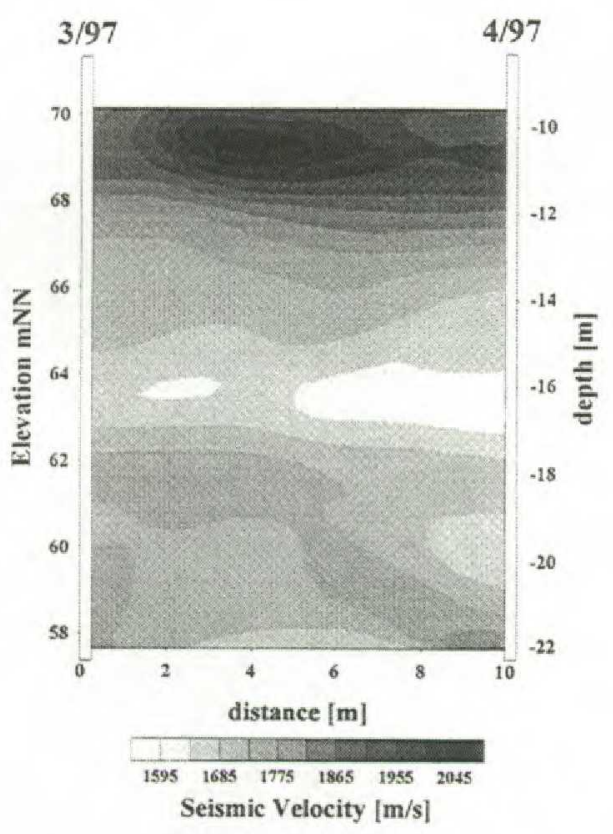

Fig. 2: Velocity tomogram 3/97 - 4/97

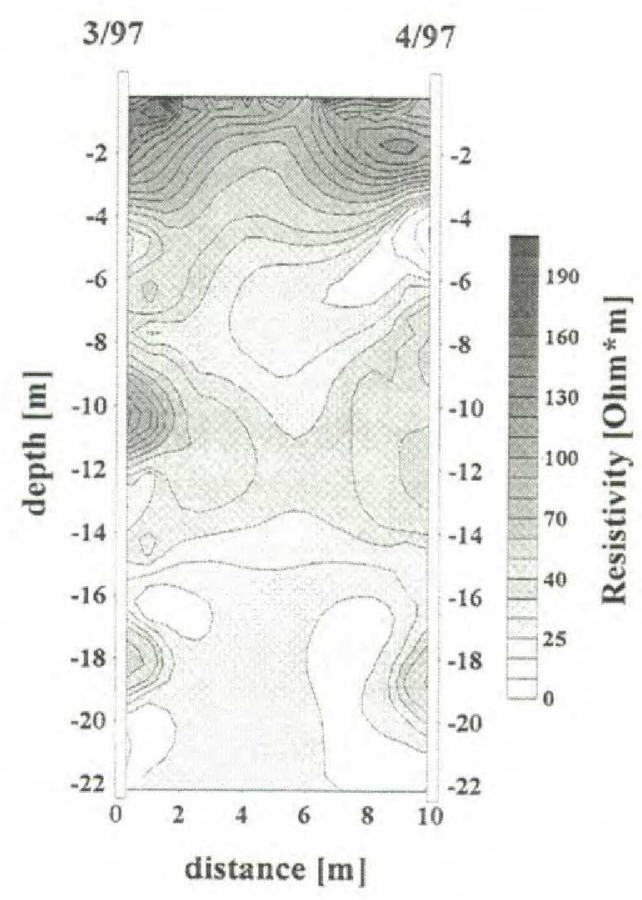

Fig. 3: Resistivity tomogram 3/97 - 4/97

From the resistivity tomogram the subsurface can be divided into four zones, see fig. 3 . On the surface (top of tomogram) a zone of high resistivity with a thickness of about $4 \mathrm{~m}$ is shown. From $-4 \mathrm{~m}$ to $-10 \mathrm{~m}$ a zone having a low resistivity of $40 \Omega \mathrm{m}$ can be observed. Below a depth of $-10 \mathrm{~m}$ a high resistivity zone is recovered which corresponds to the high velocity zone seen in fig. 2. At a depth of $-13 \mathrm{~m}$ the tomogram shows predominantly low resistivities up to $50 \Omega \mathrm{m}$. As seen, the resolution within the resistivity tomogram is lower compared to the seismic tomogram. To calculate the final lithogram both data sets had to be interpolated on the same grid from a depth of $-10 \mathrm{~m}$ to $-22 \mathrm{~m}$. This was achieved by conditional kriging. At each grid point a parameter set of seismic velocity and electric was obtained. From the borehole data information about the sediments was obtained indicating four main groups: fine sand, coarse sand, coarse sand to gravel, gravel. Based on the drilling and borehole information a subdivision of the tomograms into four groups or clusters was assumed. To invert both tomograms and to derive a combined tomogram k-means cluster analysis was applied to the parameter set [6]. The k-means clustering minimizes the overall variance between the parameters and their cluster means. The clustering routine iterates from 1 to the desired number of clusters. The first iteration starts with one cluster for all grid points and the summary statistic is computed for all distances from each grid point to the cluster centre. The second iteration divides the cluster into two clusters by moving the grid point data from one to the other cluster until no further movement decreases the distances between each grid point to its cluster centre. For all subsequent iterations, the cluster with the largest variance is split and its grid point parameters are assigned to the cluster whose mean is the shortest distance from the grid point. The means are then updated and the clustering process is continued until no reassignments are made for an iteration. The clustering results in a tomogram subdivided into the desired numbers of clusters. To each grid point a cluster is assigned having a mean value and a variance. The resulting lithogram is shown in figure 4. 


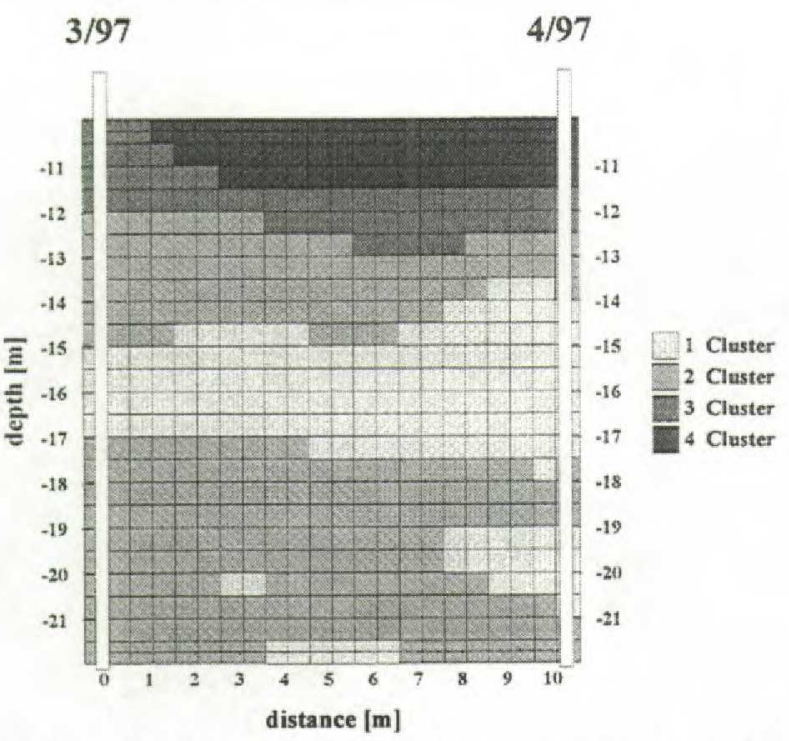

Fig. 4: Lithogram obtained from cluster analysis

\section{Conclusions}

Seismic and electric tomography was carried out at a test-site near Bitterfeld, Germany. Both tomographic methods were combined to achieve a joint inversion and to map the sedimentological situation at the site. The inversion was carried out using a cluster algorithm. The use of four clusters showed a distinct subdivision into different lithological zones. Using the borehole data sedimentological classes can be assigned to each cluster. The sediments of the cluster 1 were found to be predominantly fine sandy materials. Cluster 2 consisted of fine up to coarse sandy sediments, whereas cluster 3 was composed of coarse sandy up to gravel sediments. Coarse sandy sediments formed cluster 4 . The cluster results are given in table 1 .

\begin{tabular}{|c|c|c|}
\hline Cluster/Parameter & Seismic velocity $[\mathrm{m} / \mathrm{s}]$ & Electric resisitvity $[\Omega \mathrm{m}]$ \\
\hline 1 & 1657 & 28 \\
\hline 2 & 1728 & 31 \\
\hline 3 & 1853 & 54 \\
\hline 4 & 1978 & 43 \\
\hline
\end{tabular}

Tab. 1: Results of cluster analysis with cluster means

Acknowledgement: We would like to acknowledge Umweltforschungszentrum Halle-Leipzig $\mathrm{GmbH}$ for their financial and technical support.

\section{References}

[1] Hyndman, D. , Harris, J. and Gorelik, S. (1994): Coupled seismic and tracer test inversion for aquifer characterization, WRR, 30, No. 7, p. 1965-1977.

[2] Yamamoto, T., Nye, Th. und Kuru, M. (1995): Imaging the permeability structure of a limestone aquifer by crosswell acoustic tomography, Geophysics, Vol. 60, No. 6, p. 1634-1645.

[3] Marion, D. (1990): Acoustical, mechanical and transport properties of sediments and granular materials, Dissertation, Stanford University, Californien.

[4] Copty, N., Rubin, Y. und Mavko, G. (1993): Geophysical-hydrological identification of field peremabilities through bayesian updating, WRR, Vol. 29, No. 8, p. 2813-2825.

[5] Lörtzer, G.J.M. and Berkhout A.J. (1992): An integrated approach to lithologic inversion - Part I: Theory, Geophysics, Vol. 57, No. 2, p.233-244.

[6] IMSL (1987): FORTRAN Subroutines for statistical analysis, IMSL Inc. Houston, USA, Version 1.0.

[7] Dietrich, P. \& Fechner, Th. (1996): Using sensitivity analysis for the planning and interpretation of geoelectrical tracer experiments, II. EEGS meeting, Nantes, p. 308-311. 